\title{
Natural Deep Eutectic Solvents as a New Extraction Media for Phenolic Metabolites in Carthamus tinctorius L.
}

\author{
Yuntao Dai, ${ }^{\dagger}$ Geert-Jan Witkamp ${ }^{\ddagger}$ Robert Verpoorte, ${ }^{\dagger}$ and Young Hae Choi ${ }^{*}{ }^{\dagger}$ \\ ${ }^{\dagger}$ Natural Products Laboratory, Institute of Biology, Leiden University, 2300 RA Leiden, The Netherlands \\ ${ }^{\star}$ Department of Biotechnology, Delft University of Technology, Delft, The Netherlands
}

\section{Supporting Information}

ABSTRACT: Developing green solvents with low toxicity and cost is an important issue for the biochemical industry. Synthetic ionic liquids and deep eutectic solvents have received considerable attention due to their negligible volatility at room temperature, high solubilization ability, and tunable selectivity. However, the potential toxicity of the synthetic ionic liquids and the solid state at room temperature of most deep eutectic solvents hamper their application as extraction solvents. In this study, a wide range of recently discovered natural ionic liquids and deep eutectic solvents (NADES) composed of natural compounds were investigated for the extraction of phenolic compounds of diverse polarity. Safflower was selected as a case study because its aromatic pigments cover a wide range of polarities. Many advantageous features of NADES (such as their sustainability, biodegradability combined with

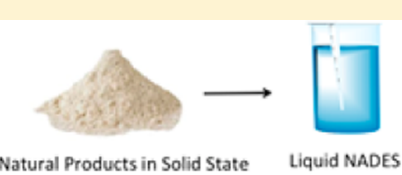

Natural Products in Solid State Liquid NADES

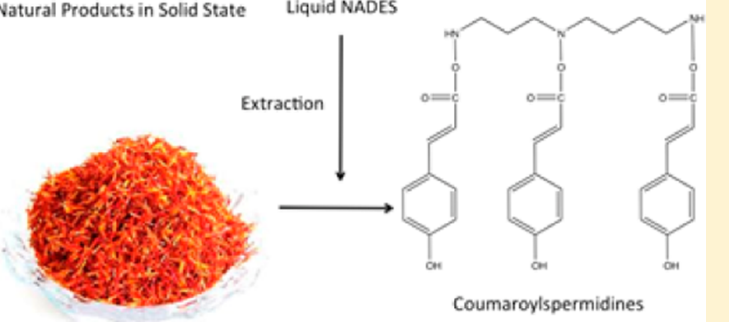
acceptable pharmaceutical toxicity profiles, and their high solubilization power of both polar and nonpolar compounds) suggest their potential as green solvents for extraction. Experiments with different NADES and multivariate data analysis demonstrated that the extractability of both polar and less polar metabolites was greater with NADES than conventional solvents. The water content in NADES proved to have the biggest effect on the yield of phenolic compounds. Most major phenolic compounds were recovered from NADES with a yield between $75 \%$ and $97 \%$. This study reveals the potential of NADES for applications involving the extraction of bioactive compounds from natural sources.

Con onventional organic solvents are widely used in the preparation of bioactive components from natural product resources in the pharmaceutical, food, and cosmetic industry. The wide range of polarity and physical properties of natural compounds makes the extraction of all metabolites from biomass in a one-step process with one single solvent virtually impossible. ${ }^{1}$ Thus, a wide range of solvents of different polarities is required for the extraction, separation, and purification as well as administration to humans of various drugs. So far, alcohols, chloroform, and ethyl acetate are used for these purposes. However, the use of large amounts of organic solvents can pollute the environment and leave unacceptable residues of organic solvents in extracts. ${ }^{2}$

With the aim of developing environmentally friendly solvents, ionic liquids (ILs) have received increasing attention because they possess attractive properties such as negligible volatility at room temperature ${ }^{3,4}$ and adjustable physicochemical properties ${ }^{5}$ that give the ability to dissolve a wide range of solutes and allow for tailor-made selectivities for extractions and separations. ${ }^{6,7}$ Compared with molecular liquids, ILs are a class of organic salts with a low melting point $\left(<100{ }^{\circ} \mathrm{C}\right)$. Synthetic ILs have mainly been used in the field of organic chemistry, ${ }^{3}$ electrochemistry, ${ }^{8}$ analytical chemistry, ${ }^{9}$ and chemical engineering. ${ }^{10}$ However, the use of synthetic ILs as solvents for extraction in the pharmaceutical industry is limited because of the high toxicity of some of their ingredients, ${ }^{11,12}$ their irritating properties, and high cost of the synthesis of their components. Ionic liquids have been used to extract some active compounds from plant material. ${ }^{13-17}$

Deep eutectic solvents $(\mathrm{DES})^{18,19}$ are another type of solvents with the similar physical properties of ILs. These solvents are composed of a mixture of organic compounds and have a melting point that is far below that of either individual component. A number of DES made up of different components, such as choline, urea, ${ }^{19}$ organic acids, ${ }^{20}$ and sugars, have been reported. ${ }^{21}$ Compared with ILs, DES show some advantages as solvents, especially considering their lower environmental and economic impact, e.g., biodegradability, pharmaceutical acceptable toxicity, low cost, and simple preparation methods. They have been used as solvents to extract DNA and as media for enzymatic reactions. ${ }^{22,23}$ However, the high viscosity and solid state of most DES at room temperature restrict their application as extraction solvents ${ }^{19-21}$ and there is still no report on their use for obtaining active compounds from plant materials.

Received: February 8, 2013

Accepted: May 27, 2013

Published: May 27, 2013 
In previous studies, we developed a series of natural ionic liquids and deep eutectic solvents composed of primary metabolites common in living cells, named natural deep eutectic solvents (NADES); ${ }^{24}$ they may sometimes include water among their ingredients. In certain molar ratios (e.g., equimolar), these NADES show strong intermolecular interactions. Apart from sharing advantages of reported ILs and DES, NADES possess better properties for extraction, e.g., liquid state even below $0{ }^{\circ} \mathrm{C}$, adjustable viscosity, and sustainability. ${ }^{25}$ Natural deep eutectic solvents contain components that are abundant in our daily food, being thus cheap, sustainable, and safe. Interestingly, some NADES show a very high solubilization ability of both nonpolar and polar compounds, and some metabolites are significantly more soluble in NADES than in water. ${ }^{24,25}$ Moreover, they have proved to be able to dissolve even macromolecules. ${ }^{25,26}$ This predicts a great potential for NADES as solvents in the extraction of valuable secondary metabolites for their application in the food or pharmaceutical industry.

Despite the extensive research on NADES, there is still a lack of information on practical issues related to their application as an extraction solvent, such as their efficiency, optimal water content, and the recovery of extracted compounds from the NADES extracts. The latter is particularly challenging considering the inherent low vapor pressure of NADES that makes it difficult to recover solutes from the NADES solution. In this paper, we investigate three aspects of NADES as an extraction solvent: (i) the extractability of natural products of diverse polarity with NADES, (ii) the optimization of extraction parameters for these phenolic compounds with three typical NADES, and (iii) the recovery of the phenolics from the NADES extract. As an example, we selected safflower (Flos carthami), the corolla from Carthamus tinctorius L. (Asteraceae), because of its high content of phenolic compounds ${ }^{27,28}$ of a broad range of polarity and also its medicinal application for circulation disorders. It contains yellow (hydroxysafflor yellow) and red pigments. Hydroxysafflor yellow A (HSYA), the major active component of safflower, ${ }^{29,30}$ cartormin, and carthamin are the main pigments in safflower used as a dye for food and cosmetics. ${ }^{31}$ Another group of compounds present in safflower, tri-p-coumaroylspermidines, ${ }^{32,33}$ reportedly have antihuman immunodeficiency virus (HIV) and antidepressive activities. It also contains flavonoids and quercetin glycosides including rutin. ${ }^{34}$ An overview of the profiles of the NADES extracts obtained using high-performance liquid chromatography (HPLC) fingerprinting and principal components analysis (PCA) allowed us to evaluate the extractability of these compounds.

\section{EXPERIMENTAL SECTION}

Plant Material. Safflower was bought from Xinjiang province in China. The plant material was identified by one of the authors, Dr. Young Hae Choi, and a voucher specimen (NPL-carthamus-0913) was deposited in the Natural Products Laboratory, Institute of Biology, Leiden University. The dry plant material was ground into powder in a blender with liquid nitrogen.

Chemicals and Reagents. Ethanol of analytical grade and acetonitrile of HPLC grade were purchased from Biosolve BV (Valkenswaard, The Netherlands). Deionized water was used. Malic acid, lactic acid, proline, sucrose, glucose, fructose, 1,2propanediol, sorbitol, and choline chloride were purchased from Sigma (St. Louis, MO, U.S.A.). Macroporous resin Diaion
HP-20 from Supelco (Bellefonte, PA, U.S.A.) was used in this study. Silica gel (pore size $60 \AA$, 230-400 mesh) from SigmaAldrich (St. Louis, MO, U.S.A.) and Sephadex LH-20 from GE Healthcare Bio-Sciences (AB, Uppsala, Sweden) were used.

Natural Deep Eutectic Solvents Preparation. All NADES including lactic acid-glucose (LGH), proline-malic acid $(\mathrm{PMH})$, sucrose-choline chloride $(\mathrm{SuCH})$, glucosecholine chloride $(\mathrm{GCH})$, sorbitol-choline chloride (SoCH), 1,2-propanediol-choline chloride $(\mathrm{PCH})$, and fructoseglucose-sucrose (FGSH) were prepared according to our previously reported method. ${ }^{25}$

Extraction with Different Solvents. Extraction was performed in a sealed bottle with $100 \mathrm{mg}$ of plant material and $1.5 \mathrm{~mL}$ of solvent, heating and stirring at $40^{\circ} \mathrm{C}$ for $1 \mathrm{~h}$. The sample was transferred to a $2 \mathrm{~mL}$ microtube and centrifuged at $10968 \mathrm{~g}$ for $20 \mathrm{~min}$. The suspension was then filtered through a $0.45 \mu \mathrm{m}$ cellulose acetate filter and diluted with the same volume of water. Each extraction was performed by triplicate.

Extraction Parameter Optimization. The extraction parameters of the NADES SuCH, LGH, and PMH were optimized. These included the ratio between plant material weight and NADES volume $(\mathrm{mg} / \mathrm{mL})(40: 1,30: 1,20: 1$, and $10: 1)$, the water content in NADES $(0 \%, 10 \%, 25 \%, 50 \%$, and $75 \%)$, and the extraction time $(30,60,90,120$, and $180 \mathrm{~min})$.

Recovery of Compounds from NADES Extracts. Samples of $400 \mathrm{mg}$ of powdered plant material were extracted with $6 \mathrm{~mL}$ of $\mathrm{SuCH}, \mathrm{PMH}$, and $40 \%$ ethanol using the abovedescribed conditions. The obtained extract was divided into two parts, one for HPLC-DAD analysis as the reference, and one for recovery tests of phenolic compounds from the NADES extract. For the recovery test the $\mathrm{SuCH}$ and $\mathrm{PMH}$ extracts were submitted to the following procedure: $1 \mathrm{~mL}$ of the extract was diluted with $10 \mathrm{~mL}$ of deionized water (for $\mathrm{SuCH}$ extract, $1 \%$ formic acid was added), loaded on an HP-20 column of $60 \mathrm{~g}$ (height $50 \mathrm{~cm}$ ), and eluted with sufficient deionized water (for $\mathrm{SuCH}$ extract, $1 \%$ formic acid was added) until all the NADES was washed out. The sample was then eluted with $130 \mathrm{~mL}$ of $50 \%$ ethanol and $260 \mathrm{~mL}$ of ethanol. These two ethanolic fractions were combined, dried with a vacuum evaporator, and redissolved with $3 \mathrm{~mL}$ of $50 \%$ methanol. An aliquot of $1 \mathrm{~mL}$ of the diluted solution was analyzed with HPLC-DAD and compared with the equally diluted initial extract. Another 2 $\mathrm{mL}$ of the extract were dried, redissolved in $0.4 \mathrm{~mL}$ of methanol- $d_{4}$ (99.80\% from Cambridge Isotope Laboratories, Andover, MA) and $0.4 \mathrm{~mL}$ phosphate buffer $\left(\mathrm{KH}_{2} \mathrm{PO}_{4}, \mathrm{pH} 6.0\right)$ in deuterium oxide (CortecNet, Voisins-Le-Bretonneux, France), and analyzed by ${ }^{1} \mathrm{H}$ NMR together with $40 \%$ ethanol extract with the same dilution. The above experiments were performed by triplicate.

Isolation of Pure Compounds. A sample of $100 \mathrm{~g}$ of dry ground material was sonicated with two $800 \mathrm{~mL}$ portions of methanol for $1 \mathrm{~h}$, filtered, and dried with a rotary evaporator. The residue $(20.7 \mathrm{~g})$ was partitioned with $90 \%$ methanol and $n$ hexane twice, and the aqueous methanolic fraction was evaporated under vacuum, obtaining a residue of $18 \mathrm{~g}$. This was fractionated on a middle pressure column with $180 \mathrm{~g}$ of silica gel and eluted with $500 \mathrm{~mL}$ of $n$-hexane-chloroform (1:1), $1000 \mathrm{~mL}$ of chloroform, $500 \mathrm{~mL}$ of chloroformmethanol (10:1), and $2000 \mathrm{~mL}$ of methanol. Each fraction (100 $\mathrm{mL}$ ) was pooled based on its thin-layer chromatography (TLC) profile [stationary phase, $60 \mathrm{~F}_{254}$ plate (Merk, Darmstadt, Germany); mobile phase, chloroform-MeOH (8.5:1.5)]. Three fractions containing phenolic compounds-26 (40 


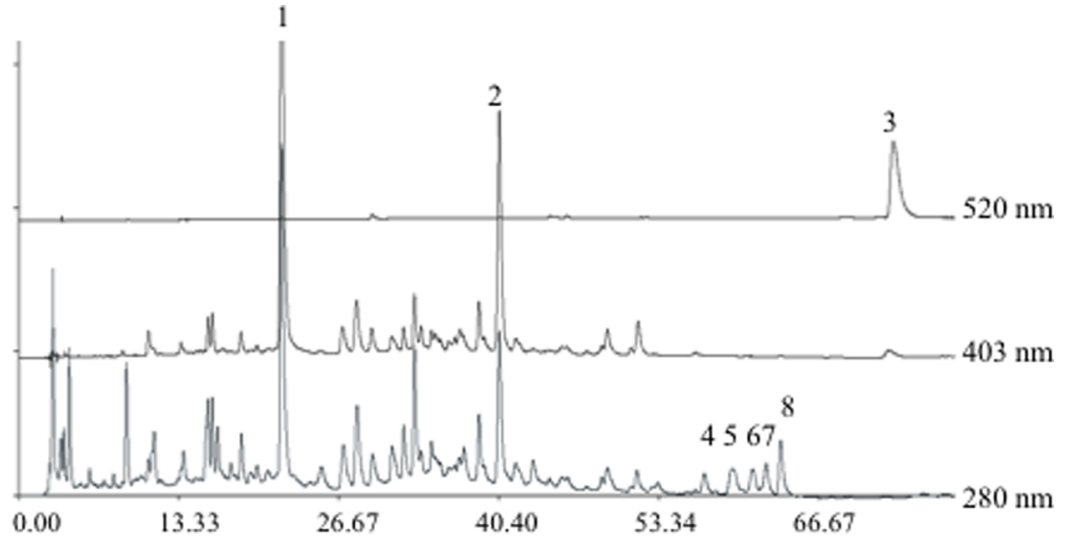

Figure 1. HPLC-DAD chromatographic profiles of the extract in natural deep eutectic solvents (NADES) from safflower at three different wavelengths (the labeled compounds are as follows: 1, hydroxysafflor yellow A; 2, cartormin; 3, carthamin; 4, $N^{1}, N^{10}, N^{5}-(Z)$-tri- $p$ coumaroylspermidine; 5, $N^{1}$ - $(E)-N^{5}, N^{10}$ - $(Z)$-tri-p-coumaroylspermidine; 6, $N^{1}, N^{10}-(E)$ - $N^{5}$ - $(Z)$-tri-p-coumaroylspermidine; 7, stereoisomer of tri-pcoumaroylspermidine; $8, N^{1}, N^{10}, N^{5}-(E)$-tri- $p$-coumaroylspermidine).

$\mathrm{mg}), 29-30$ (56 mg), 33-37 (127 mg) - were collected. Each combined fraction was purified on a column with $55 \mathrm{~g}$ of Sephadex LH-20, eluted with methanol, and further purified with semipreparative HPLC, using a Phenomenex Luna C18 (250 mm $\times 10 \mathrm{~mm}, 5 \mu \mathrm{m}$, Torrance, CA, U.S.A.) column and acetonitrile-water $(23: 77,3 \mathrm{~mL} / \mathrm{min})$ as a mobile phase. The isolated compounds were dissolved in $1.0 \mathrm{~mL}$ of methanol- $d_{4}$ for measurement for their structural elucidation.

HPLC, NMR, and MS Analysis. Quantitative HPLC analysis was performed on an Agilent 1200 chromatographic system with a photodiode array detector (DAD) and separated on an HPLC column, Phenomenex Luna C18 $(4.6 \mu \mathrm{m} \times 250$ $\mathrm{mm}, 5 \mu \mathrm{m})$. The mobile phase consisted of water with $0.5 \%$ $\mathrm{H}_{3} \mathrm{PO}_{4}(\mathrm{~A})$ and acetonitrile (B) in a linear gradient program as follows: $5-11 \% \mathrm{~B}(0-10 \mathrm{~min}), 11-14 \% \mathrm{~B}(10-16 \mathrm{~min}), 14 \%$ B (16-23 min), 14-20\% B (23-30 min), 20-35\% B (30-70 $\mathrm{min}), 35-60 \% \mathrm{~B}(70-80 \mathrm{~min})$ at a flow rate of $1.0 \mathrm{~mL} / \mathrm{min}^{34}$ Chromatograms were recorded at 520, 403, and $280 \mathrm{~nm}$. The injection volume was $10 \mu \mathrm{L}$.

${ }^{1} \mathrm{H}$ NMR spectra, correlation spectroscopy (COSY), Jresolved spectra, heteronuclear single-quantum coherence (HSQC), heteronuclear multibond correlation spectroscopy (HMBC), and attached proton test ${ }^{13} \mathrm{C}$ NMR (APT) of four purified compounds were recorded at $25{ }^{\circ} \mathrm{C}$ on a $600 \mathrm{MHz}$ Bruker DMX-600 spectrometer (Bruker, Karlsruhe, Germany) operating at a proton NMR frequency of $600.13 \mathrm{MHz}\left({ }^{1} \mathrm{H}\right)$ and $150.13 \mathrm{MHz}\left({ }^{13} \mathrm{C}\right)$ with $\mathrm{MeOH}-d_{4}$ as the internal lock. All the parameters followed those described in our previous report. ${ }^{35}$

Mass spectra were measured by an electrospray ionization time-of-flight mass spectrometer (ESI-TOF-MS). The operating conditions of the ESI ion source (Jeol, Tokyo, Japan) coupled to a JMS-T100TD (AccuTOF-TLC) in the positive ion modes were a discharge needle voltage of $2000 \mathrm{~V}$ and nebulizing nitrogen gas flow at $1 \mathrm{~L} / \mathrm{min}$. The first orifice lens was set to $100 \mathrm{~V}$, and ring lens voltage was set to $13 \mathrm{~V}$. The TOF-MS was set with a peak voltage of $2500 \mathrm{~V}$, a bias voltage of $29 \mathrm{~V}$, a pusher bias voltage of $-0.76 \mathrm{~V}$, and a detector voltage of $2300 \mathrm{~V}$.

Data Analysis. The areas of eight representative peaks in the HPLC-DAD chromatograms from triplicates were subjected to PCA with the Pareto scaling method using the SIMCA-P software (version 12.1, Umetrics, Umeå, Sweden). These peaks corresponded to compounds of the whole range of polarities present in the HPLC-DAD chromatograms. Analysis of variance (ANOVA) was performed in SPSS software (version 14.0, Chicago, IL, U.S.A.) using the area of peaks in the HPLC chromatogram, and $P$ values $\leq 0.1$ were considered as significant for comparison. The relative extraction yield was calculated on the basis of the peak area of the selected peak in the HPLC chromatograms of initial extracts. The recovery yield ( $\mathrm{w} \%$ ) was calculated with the peak area of selected peaks in HPLC chromatograms as follows:

$$
\mathrm{w} \%=\left(A_{\mathrm{rec}} / A\right) \times 100 \%
$$

where $A_{\text {rec }}$ is the peak area of a compound in the chromatogram of the recovered mixture and $A$ is the peak area of the same compound in the chromatogram of the initial extract.

\section{RESULTS AND DISCUSSION}

Comparison of the Extractability of Safflower Polyphenols with NADES, Water, and Ethanol. Seven NADES with different polarity, viscosity, composition, and solubilization abilities were selected in this study to test their extraction efficiency. These were LGH, PMH, SuCH, GCH, SoCH, PCH, and FGSH. Physical properties of NADES differ according to their composition ${ }^{25}$ as does their solubilizing ability of diverse natural products. ${ }^{24,25}$

The major disadvantage of NADES when compared with conventional solvents is their inherent high viscosity. Viscosity is known to hinder the efficiency of NADES as extraction solvents since it results in a slow mass transfer. To solve the problem, extraction conditions were adjusted to reduce the viscosity of NADES and improve the yield. The viscosity of NADES differs enormously according to their composition, but in all cases it can be reduced by the addition of a certain amount of water. ${ }^{25}$ Thus, 75\% SuCH, 75\% FGSH, 75\% PMH, $90 \% \mathrm{GCH}$ solutions in water (v/v) were used in this study. Another variable known to affect viscosity is temperature, and using $40{ }^{\circ} \mathrm{C}$ as the extraction temperature resulted in a decrease in viscosity and increased yields. ${ }^{25}$ Lastly, in an effort to increase the transport rate of the compounds in the liquid with an external force, mechanical agitation was used instead of ultrasound. This resulted in a higher efficiency. Thus, phenolic metabolites were extracted from safflower by agitation of 100 mg of plant material in $1.5 \mathrm{~mL}$ of solvent at $40^{\circ} \mathrm{C}$ as described 
<smiles>O=C(/C=C/c1ccc(O)cc1)C1=C(O)C(O)(C(Cl)(Cl)Cl)C(O)=C(Cl)C1=O</smiles>

1

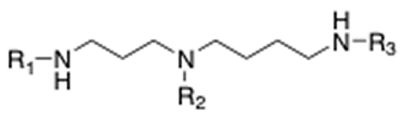

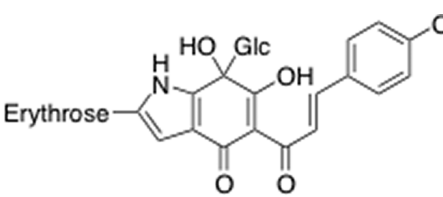

2

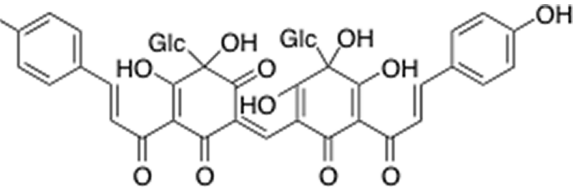

3

$\mathrm{R}_{1}=\mathrm{R}_{2}=\mathrm{R}_{3}=$ cis-p-coumaroyl, 4

$\mathrm{R}_{1}=$ trans-p-coumaroyl, $\mathrm{R}_{2}=\mathrm{R}_{3}=$ cis- $p$-coumaroyl, 5

$\mathrm{R}_{1}=\mathrm{R}_{3}=$ trans- $p$-coumaroyl, $\mathrm{R}_{2}=$ cis- - -coumaroyl, 6

$\mathrm{R}_{1}=\mathrm{R}_{2}=\mathrm{R}_{3}=$ cis/trans- - -coumaroyl, 7

$\mathrm{R}_{1}=\mathrm{R}_{2}=\mathrm{R}_{3}=$ trans- - -coumaroyl, 8

Figure 2. Chemical structures of target phenolic compounds $\mathbf{1 - 8}$ in safflower.

in the Experimental Section. Given the high viscosity of the resulting extracts the samples were centrifuged before filtration.

Clear differences in the extractability of compounds from safflower with the tested NADES, water, and ethanol were reflected in the color of the obtained extracts and their HPLC profiles (Supporting Information Figure S-1). The NADES extracts exhibited the most intense color (Supporting Information Figure S-1a). The HPLC fingerprints of NADES extracts showed all the peaks observed in the aqueous and ethanol extracts (Supporting Information Figure S-1b, Figure 1). Three NADES, LGH, PMH, and $\mathrm{SuCH}$, extracted relatively polar compounds, HSYA $(\mathrm{Tr}=21.9 \mathrm{~min})^{29,34}$ and cartormin $(\mathrm{Tr}=40.0 \mathrm{~min}),{ }^{29,36}$ and less polar compounds, carthamin $(\mathrm{Tr}$ $=72.9 \mathrm{~min}$, identified by comparison with a reference compound) and five stereoisomers of tri- $p$-coumaroylspermidine $(\operatorname{Tr}=57.1-63.5 \mathrm{~min})$. The identification of the compounds (Figure 1) corresponding to each peak was confirmed by comparing NMR, HRESI-MS data obtained by UPLD-ESI-TOF-MS, previous papers, and 2D NMR spectra. $^{32,33,37}$ Compounds corresponding to four peaks were isolated and identified as $N^{1}, N^{10}, N^{5}-(Z)$-tri-p-coumaroylspermidine (4), $N^{1}-(E)-N^{5}, N^{10}-(Z)$-tri-p-coumaroylspermidine (5), $N^{1}, N^{10}-(E)-N^{5}-(Z)$-tri-p-coumaroylspermidine (6), and $N^{1}, N^{10}, N^{5}-(E)$-tri-p-coumaroylspermidine (8) (Figure 2). The compound 7 (a mixture of two compounds) and a small peak between the compounds $\mathbf{4}$ and $\mathbf{5}$ in HPLC chromatogram all have the same UV spectrum with a UV max at around $300 \mathrm{~nm}$ and the same molecular weight as the above-described tri-pcoumaroylspermidines, probably corresponding thus to other stereoisomers of tri- $p$-coumaroylspermidine. The ${ }^{1} \mathrm{H}$ and ${ }^{13} \mathrm{C}$ NMR spectra of the coumaroylspermidine showed complex signals because of the restricted rotation around the $\mathrm{N}-\mathrm{C}\left(\mathrm{sp}^{2}\right)$ bond in the $p$-coumaroylspermidine. ${ }^{38,39}$

The retention times in the HPLC profile and relative extraction ratio of those compounds in water and ethanol extracts corresponded well with their polarity. Tri- $p$-coumaroylspermidines and carthamin were highly extractable in all three NADES and ethanol, while water did not extract these less polar compounds. Moreover, LGH, $\mathrm{PMH}, \mathrm{SuCH}$ were more efficient than ethanol even for the less polar compound, carthamin. The chemical profile of some NADES extracts was qualitatively similar to that of a $40 \%(\mathrm{v} / \mathrm{v})$ ethanol extract that had been reported to be the optimal solvent for extracting safflower yellow. ${ }^{40}$

To investigate the difference between the efficiency of NADES and conventional solvents on one hand, and among the selected NADES on the other, multivariate data analysis was applied using the peak areas of the different HPLC peaks as variables. There are numerous peaks in the UV trace, including those visible at different wavelengths $(280,520$, and $403 \mathrm{~nm})$ (Figure 1). Because of the complexity caused by overlapping and minor compounds, eight peaks at different retention times were selected as the variables for assessing the efficiency of different solvents. The eight representative peaks included the two largest peaks observed in the $403 \mathrm{~nm}$ chromatogram (HSYA and cartormin), the major peak in the $520 \mathrm{~nm}$ trace (carthamin), and five characteristic peaks at $280 \mathrm{~nm}$ (five stereoisomers of tri-p-coumaroylspermidine). The chosen compounds represent the whole range of polarity and include major and important active metabolites in the safflower extract. They should thus reflect the extraction efficiency of NADES in terms of polarity range, selectivity, and even activity.

The score plot of PCA of the first two components $\left(R^{2}=\right.$ 0.94 and $Q^{2}=0.89$ ) (Figure 3a) showed a separation of the extracts into four groups. The PCA confirmed the similarity between the $\mathrm{LGH}, \mathrm{PMH}, \mathrm{SuCH}$, and the $40 \%$ ethanol extracts (group I), whereas the $\mathrm{PCH}$ extract was similar to the ethanol extract (group II). In the loading plot of PCA, all metabolites selected from the chromatograms were clustered around group I (Figure 3b), confirming that solvents in group I had a broad extraction capacity, being efficient for the extraction of polar compounds (HSYA and cartormin) and less polar ones (carthamin and five stereoisomers of tri- $p$-coumaroylspermidine). In addition, solvents in group II ( $\mathrm{PCH}, \mathrm{EtOH})$ were efficient in extracting less polar compounds (carthamin and tri$p$-coumaroylspermidines), while those in group IV (FGSH and water) exhibited a high efficiency for polar ones (HSYA and cartormin), which is in agreement with reports on the efficiency of water to extract the yellow pigment from safflower. ${ }^{41}$ Therefore, the extractability of phenolic compounds from safflower proved to be higher in $\mathrm{SuCH}, \mathrm{PMH}$, and LGH.

Optimization of the Extraction Parameters for NADES with High Extractability ( $\mathrm{SuCH}, \mathrm{PMH}, \mathrm{LGH})$. Extraction parameters were optimized using $\mathrm{SuCH}, \mathrm{PMH}$, and $\mathrm{LGH}$ as extraction solvents. The peak areas of HSYA, cartormin, and carthamin were used as the compounds to evaluate their extractability. The areas corresponding to peaks of the five tri- $p$ coumaroylspermidines were deleted because they had a retention time close to carthamin and were very small.

The water content in NADES had a great effect on their extract yield, varying considerably according to the target compounds and the NADES itself (Figure $4 a-c$ ). In the case of HSYA and cartormin, the highest extraction yield was achieved with $50-100 \%$ water in $\mathrm{SuCH}$, with $25-50 \%$ water in $\mathrm{PMH}$, 
a)
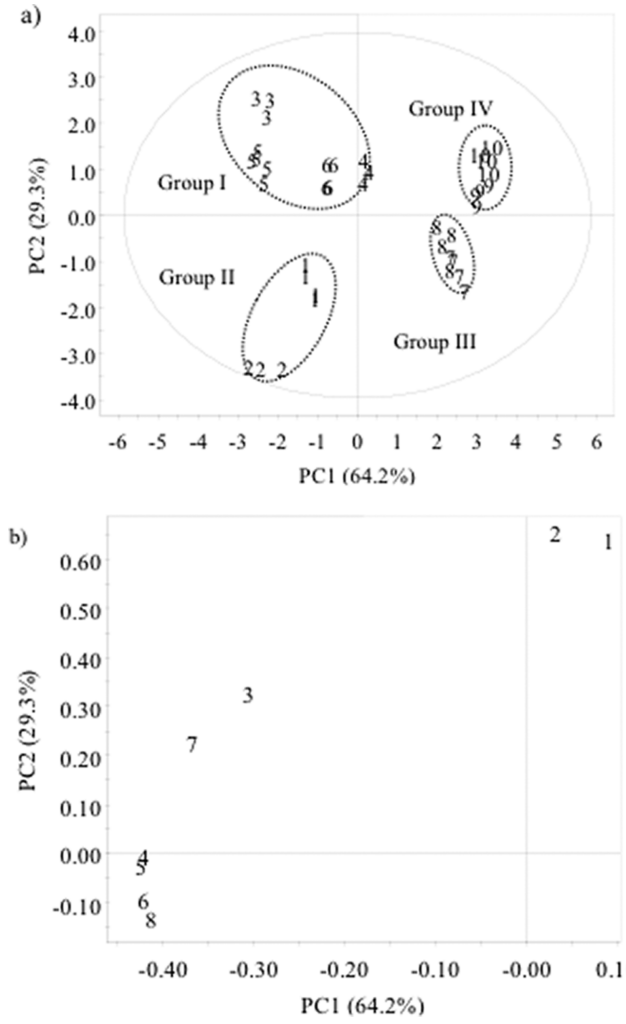

Figure 3. Score plot (a) and loading plot (b) of principal component analysis of the extracts from safflower with different solvents. (In the score plot (a): 1, 1,2-propanediol-choline chloride; 2, ethanol; 3, 40\% ethanol; 4, sucrose-choline chloride; 5, lactic acid-glucose; 6, proline-malic acid; 7, glucose-choline chloride; 8, sorbitol-choline chloride; 9, fructose-glucose-sucrose; 10, water. In the loading plot (b), the number refers to the same compounds as that in Figure 1.)

and with no addition of water in LGH. For less polar compounds, such as carthamin, the extraction yield was also greatly affected by the water content in the NADES. The highest extraction yield of carthamin was reached with $25 \%$ (v/ v) water in $\mathrm{SuCH}, 10 \%$ water in $\mathrm{PMH}$, and no water in $\mathrm{LGH}$. The extraction yield of carthamin in $\mathrm{SuCH}$ with $25 \%$ water was around 3 times higher than in $\mathrm{SuCH}$ with $10 \%$ or $50 \%$ water. So, in general, it is possible to conclude that NADES with a high water content performed better for polar compounds while NADES with low water content are suitable for the extraction of less polar compounds. The extraction yield was not significantly affected by any of the other two studied factors: extraction time and ratio of material weight to solvent volume (Supporting Information Figure S-2).

Thus, the optimized extraction conditions for these NADES were established as the following: $1 \mathrm{~h}$; ratio between material weight and solvent $30 \mathrm{mg} / \mathrm{mL}$. Under these conditions, the relative extract yield of $75 \% \mathrm{PMH}, 75 \% \mathrm{SuCH}$, and $\mathrm{LGH}$ was compared with that of ethanol, $40 \%$ ethanol, and water (Table 1) The most efficient extraction solvent proved to be $\mathrm{PMH}$ (75\%) for HSYA (similar to water and 8\% higher than $40 \%$ ethanol) and cartormin (14\% higher than water and $40 \%$ ethanol). In the case of carthamin, LGH showed the highest extraction yield (23\% higher than $40 \%$ ethanol).

Recovery Test of Phenolic Compounds from NADES. In order to recover the phenolic compounds from NADES extracts, a chromatographic resin, Diaion HP-20, was used. This resin can adsorb phenolic compounds while the polar

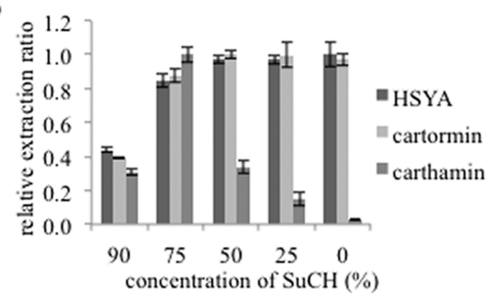

b)
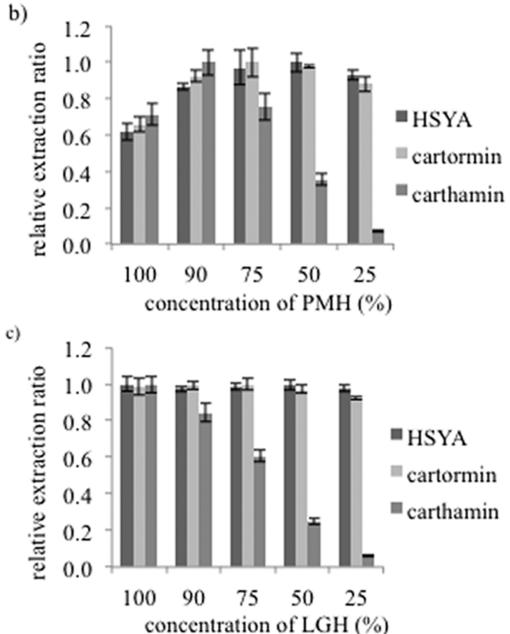

Figure 4. Effect of water content in NADES on the extraction ability of (a) sucrose-choline chloride $(\mathrm{SuCH}),(\mathrm{b})$ proline-malic acid $(\mathrm{PMH})$, and (c) lactic acid-glucose (LGH). The extraction ability is expressed as a relative extraction ratio in which the value of the peak area of a certain compound is divided by the highest peak area of the same compound obtained from different concentrations of water in that same NADES.

Table 1. Relative Extraction Yield of Three Representative Metabolites [Hydroxysafflor Yellow A (HSYA), Cartormin, and Carthamin] from Safflower with Six Different Solvents ${ }^{a}$

\begin{tabular}{lccc} 
& \multicolumn{2}{c}{ relative extraction yield as peak area } \\
\cline { 2 - 4 } \multicolumn{1}{c}{ solvents } & HSYA & cartormin & carthamin \\
$75 \% \mathrm{PMH}^{b}$ & $2813 \pm 2$ & $2925 \pm 37$ & $134 \pm 0$ \\
$75 \% \mathrm{SuCH}^{b}$ & $2680 \pm 3$ & $2591 \pm 9$ & $152 \pm 0$ \\
$\mathrm{LGH}^{b}$ & $2244 \pm 123$ & $2229 \pm 54$ & $235 \pm 26$ \\
$40 \% \mathrm{EtOH}$ & $2611 \pm 80$ & $2528 \pm 79$ & $182 \pm 42$ \\
water & $2843 \pm 28$ & $2520 \pm 19$ & $5 \pm 1$ \\
ethanol & $30 \pm 0$ & $13 \pm 1$ & $12 \pm 0$
\end{tabular}

${ }^{a}$ The data correspond to the area of each peak in the HPLC-DAD chromatogram at $403 \mathrm{~nm}$ for HSYA and cartormin, and $520 \mathrm{~nm}$ for carthamin, obtained with the injection volume of $10 \mu \mathrm{L}$ of the extract prepared by treatment of $90 \mathrm{mg}$ of safflower powder with $3 \mathrm{~mL}$ of solvent and dilution to twice its volume with water. The data are expressed in mean \pm SD based on triplicate. ${ }^{b} 75 \% \mathrm{PMH}, 75 \%$ (v/v) proline-malic acid in water; $75 \% \mathrm{SuCH}, 75 \%(\mathrm{v} / \mathrm{v})$ sucrose-choline chloride in water; LGH, lactic acid-glucose.

ingredients of NADES can be eluted with water. Most phenolic compounds were recovered with ethanol after eluting the polar compounds with water. However, the presence of components of NADES-highly concentrated aqueous solutions of the ingredients, as the case in $\mathrm{SuCH}$-affected the separation process. Using water, phenolic compounds were eluted together with sucrose from $\mathrm{SuCH}$. However, the addition of $0.1 \%$ formic acid to the water increased the retention of phenolic compounds on the column so that they were 
separated from the components of the $\mathrm{SuCH}$ solution. This did not work so well for LGH, since the separation of phenolic compounds was hindered because lactic acid was also retained on the column.

The chromatographic profiles of the solutions containing the compounds recovered from NADES (e.g., $\mathrm{PMH}$ and $\mathrm{SuCH}$ ) were qualitatively similar to the $40 \%$ ethanol extract except for the solvent peaks, as shown in the HPLC-DAD chromatogram at $280 \mathrm{~nm}$ (Supporting Information Figure S-3). Furthermore, the recovered compounds from three replicates had the same qualitative chemical profiles in HPLC-DAD chromatograms and ${ }^{1} \mathrm{H}$ NMR spectra. Recovery rates of up to $90 \%$ were achieved for polar compounds (such as HSYA and cartormin) (Table 2). In the case of the less polar compound carthamin,

Table 2. Recovery Yield of Three Representative Metabolites (Hydroxysafflor Yellow A, Cartormin, and Carthamin) in Safflower from Two NADES ${ }^{a}$

\begin{tabular}{cccc} 
& \multicolumn{3}{c}{ recovery yield (\%) } \\
\cline { 2 - 4 } NADES & HSYA & cartormin & carthamin \\
$75 \% \mathrm{PMH}^{b}$ & $92 \pm 9$ & $92 \pm 8$ & $84 \pm 8$ \\
$75 \% \mathrm{SuCH}^{b}$ & $71 \pm 4$ & $86 \pm 4$ & $90 \pm 13$
\end{tabular}

${ }^{a_{T}}$ The values are mean \pm SD based on triplicate. ${ }^{b} 75 \% \mathrm{PMH}, 75 \%$ (v/ v) proline-malic acid in water; $75 \% \mathrm{SuCH}, 75 \%(\mathrm{v} / \mathrm{v})$ sucrosecholine chloride in water.

around $84 \%$ was recovered from $\mathrm{SuCH}$ and $75 \%$ from $\mathrm{PMH}$. It is thus evident that phenolic compounds can be efficiently recovered from NADES extracts.

NADES Features Affecting Their Extraction Efficiency. The high extractability of phenolic compounds with NADES may be attributed to $\mathrm{H}$-bonding interactions between molecules of NADES and phenolic compounds. In general, the functional groups involved in H-bonds are hydroxyl, carboxylic, and amine groups, all of which are abundant in NADES while hydroxyl groups are obviously available in phenolic compounds. Our former studies demonstrated the evident interactions between quercetin and NADES. ${ }^{25}$ The phenolic compounds in safflower are $C$-glucosyl quinochalcones $^{29}$ and flavonoid glycosides. ${ }^{27}$ Therefore, H-bonding interactions between molecules of NADES and phenolic compounds are responsible for their high extractability.

The extraction capacity of NADES is also correlated with their physical properties, including polarity and viscosity. $\mathrm{PCH}$ has the lowest polarity among all the tested NADES and showed the lowest efficiency for polar compounds, such as HSYA and cartormin, but high efficiency for nonpolar compounds. Thus, the polarity of NADES has to be considered as an important property affecting its efficiency. Compared with conventional solvents, the high viscosity of NADES is an important feature, but it can be decreased by diluting it slightly with water. ${ }^{25} \mathrm{SuCH}$ is the most viscous NADES, and its viscosity is so high that it is difficult to use for the extraction of compounds from biomass. However, its dilution with water increases its efficiency, performing much better with a $25 \%$ water content than with $10 \%$ water content, most likely due to the decreasing viscosity of the aqueous $\mathrm{SuCH}$.

\section{CONCLUSIONS}

A mixture of natural solid compounds, NADES, proved to be efficient solvents for the extraction of phenolic compounds of diverse polarities. The extracted compounds were recovered from NADES with a Diaion resin column (e.g., HP-20). These simple, low-cost, green, and efficient methods can be applied to the extraction and isolation of natural products from biomaterials. This holds promise for further applications of NADES in pharmaceutical, cosmetics, and food industries for the extraction and recovery of natural products.

\section{ASSOCIATED CONTENT}

\section{Supporting Information}

Additional figures as noted in the text. This material is available free of charge via the Internet at http://pubs.acs.org.

\section{AUTHOR INFORMATION}

\section{Corresponding Author}

*E-mail: y.choi@chem.leidenuniv.nl. Fax: +31-71-527-4510.

Notes

The authors declare no competing financial interest.

\section{ACKNOWLEDGMENTS}

This work was supported by an NWO ECHO Grant (project no. 711.012.010) and partially by the Ministry of Land, Transport and Maritime Affairs (PM56641), Korea. The authors thank Professor Young Pyo Jang in the Department of Oriental Pharmaceutical Science, Colleague of Pharmacy, Kyung Hee University for UPLC-MS detection and Dr. Erica G. Wilson and Dr. Justin Fischedick at Leiden University for language revision. Yuntao Dai is thankful for the support from the Ph.D. program of the China Scholarship Council.

\section{REFERENCES}

(1) Verpoorte, R.; Choi, Y. H.; Choi, H. K. Chem. Senses 2006, 31, E67-E67.

(2) Puranik, S. B.; Sanjay Pai, P. N.; Rao, G. K. Int. J. Appl. Res. Nat. Prod. 2009, 2, 32-46.

(3) Welton, T. Chem. Rev. 1999, 99, 2071-2084.

(4) Visser, A. E.; Swatloski, R. P.; Rogers, R. D. Green Chem. 2000, 2, $1-4$.

(5) Huddleston, J. G.; Visser, A. E.; Reichert, W. M.; Willauer, H. D.; Broker, G. A.; Rogers, R. D. Green Chem. 2001, 3, 156-164.

(6) Yao, C.; Pitner, W. R.; Anderson, J. L. Anal. Chem. 2009, 81, $5054-5063$

(7) Ragonese, C.; Sciarrone, D.; Tranchida, P. Q.; Dugo, P.; Dugo, G.; Mondello, L. Anal. Chem. 2011, 83, 7947-7954.

(8) Macfarlane, D. R.; Forsyth, M.; Howlett, P. C.; Pringle, J. M.; Sun, J.; Annat, G.; Neil, W.; Izgorodina, E. I. Acc. Chem. Res. 2007, 40, $1165-1173$.

(9) Liu, M.; Lin, Z.; Lin, J. M. Anal. Chim. Acta 2010, 670, 1-10.

(10) Jenck, J. F.; Agterberg, F.; Droescher, M. J. Green Chem. 2004, 6, 544-556.

(11) Docherty, K. M.; Kulpa, C. F. Green Chem. 2005, 7, 185-189.

(12) Quijano, G.; Couvert, A.; Amrane, A.; Darracq, G.; Couriol, C.; Le Cloirec, P.; Paquin, L.; Carrie, D. Chem. Eng. J. 2011, 174, 27-32.

(13) Martín-Calero, A.; Pino, V.; Afonso, A. M. Trends Anal. Chem. 2011, 30, 1598-1619.

(14) Bica, K.; Gaertner, P.; Rogers, R. D. Green Chem. 2011, 13, 1997-1999.

(15) Lapkin, A. A.; Plucinski, P. K.; Cutler, M. J. Nat. Prod. 2006, 69, 1653-1664.

(16) Yansheng, C.; Zhida, Z.; Changping, L.; Qingshan, L.; Peifang, Y.; Welz-Biermann, U. Green Chem. 2011, 13, 666-670.

(17) Ressmann, A. K.; Strassl, K.; Gaertner, P.; Zhao, B.; Greiner, L.; Bica, K. Green Chem. 2012, 14, 940-944.

(18) Abbott, A. P.; Barron, J. C.; Ryder, K. S.; Wilson, D. Chem.Eur. J. 2007, 13, 6495-6501. 
(19) Abbott, A. P.; Boothby, D.; Capper, G.; Davies, D. L.; Rasheed,

R. K. J. Am. Chem. Soc. 2004, 126, 9142-9147.

(20) Abbott, A. P.; Capper, G.; Davies, D. L.; Rasheed, R. K.; Tambyrajah, V. Chem. Commun. 2003, 70-71.

(21) Imperato, G.; Eibler, E.; Niedermaier, J.; Konig, B. Chem. Commun. 2005, 1170-1172.

(22) Gorke, J. T.; Srienc, F.; Kazlauskas, R. J. Chem. Commun. 2008, $1235-1237$.

(23) Zhao, H.; Baker, G. A.; Holmes, S. Org. Biomol. Chem. 2011, 9, $1908-1916$.

(24) Choi, Y. H.; van Spronsen, J.; Dai, Y.; Verberne, M.; Hollmann, F.; Arends, I. W. C. E.; Witkamp, G.-J.; Verpoorte, R. Plant Physiol. 2011, 156, 1701-1705.

(25) Dai, Y.; van Spronsen, J.; Witkam, G. J.; Verpoorte, R.; Choi, Y. H. Anal. Chim. Acta 2013, 766, 61-68.

(26) Francisco, M.; van den Bruinhorst, A.; Kroon, M. C. Green Chem. 2012, 14, 2153-2157.

(27) Kazuma, K.; Takahashi, T.; Sato, K.; Takeuchi, H.; Matsumoto, T.; Okuno, T. Biosci. Biotechnol. Biochem. 2000, 64, 1588-1599.

(28) Zhou, Y. Z.; Chen, H.; Qiao, L.; Lu, X.; Hua, H. M.; Pei, Y. H. Helv. Chim. Acta 2008, 91, 1277-1285.

(29) Jin, Y.; Zhang, X. L.; Shi, H.; Xiao, Y. S.; Ke, Y. X.; Xue, X. Y.; Zhang, F. F.; Liang, X. M. Rapid Commun. Mass Spectrom. 2008, 22, $1275-1287$.

(30) Sun, L.; Yang, L.; Xu, Y. W.; Liang, H.; Han, J.; Zhao, R. J.; Cheng, Y. Brain Res. 2012, 1473, 227-235.

(31) Watanabe, T.; Hasegawa, N.; Yamamoto, A.; Nagai, S.; Terabe, S. Biosci. Biotechnol. Biochem. 1997, 61, 1179-1183.

(32) Jiang, J. S.; Lu, L.; Yang, Y. J.; Zhang, J. L.; Zhang, P. C. J. Asian Nat. Prod. Res. 2008, 10, 447-451.

(33) Zhao, G.; Gai, Y.; Chu, W. J.; Qin, G. W.; Guo, L. H. Eur. Neuropsychopharmacol. 2009, 19, 749-758.

(34) Wang, R. Q.; Yang, B.; Fu, M. H. China J. Chin. Mater. Med. 2008, 33, 2642-2646.

(35) Kim, H. K.; Choi, Y. H.; Verpoorte, R. Nat. Protoc. 2010, 5, 536-549.

(36) Yin, H. B.; He, Z. S. Tetrahedron Lett. 2000, 41, 1955-1958.

(37) Zhao, G.; Qin, G. W.; Gai, Y.; Guo, L. H. Chem. Pharm. Bull. 2010, 58, 950-952.

(38) Ma, C. M.; Nakamura, N.; Hattori, M. Chem. Pharm. Bull. 2001, 49, 915-917.

(39) Meurer, B.; Wray, V.; Wiermann, R.; Strack, D. Phytochemistry

1988, 27, 839-843.

(40) Zhang, M.; Wang, G. Z.; Liu, Y. W. China Pharm. 2009, 12, $348-349$.

(41) Zhang, F.; Zeng, B. F. Lishizhen Med. Mater. Med. Res. 2007, 18, $1720-1721$. 